\title{
Calculation of Sound Propagation in Ventilation Hole Device
}

\author{
Yuya Nishimura ${ }^{1}$, Sohei Nishimura ${ }^{2}$, Thu Lan Nguyen ${ }^{3}$, Khoa Pham Dang ${ }^{4}$ \\ ${ }^{1}$ Department of Control and Information System Engineering, National Institute of Technology \\ Kumamoto College, Japan \\ ${ }^{2}$ Department of Mechanical and Intelligent System Engineering, National Institute of Technology \\ Kumamoto College, Japan \\ ${ }^{3}$ Interdisciplinary Faculty of Science and Engineering, Shimane University, Japan \\ ${ }^{4}$ Department of Construction Technology and Organization, MienTrung University of Civil \\ Engineering
}

*Corresponding Author: Yuya Nishimura, Department of Mechanical and Intelligent System Engineering, National Institute of Technology, Kumamoto College, Japan

Received Date: 11-05-2017

Accepted Date: 16-05-2017

Published Date:19-05-2017

\begin{abstract}
The use of ventilation holes for providing healthy indoor air is commonly found in dwellings in developing countries with tropical climates. However, these ventilation holes also provide a direct path for traffic noise to enter a residence. In this study, a model of ventilation hole device with soundproofing capability was proposed. The performance of the simple ventilation hole device attached with a rectangular chamber was conducted by solving the wave equation.
\end{abstract}

Keywords: Wave equation, sound pressure, resonance frequency, higher-order mode.

\section{INTRODUCTION}

The use of ventilation holes for providing healthy indoor air is widely found in dwelling of tropical countries. The ventilation holes are commonly designed to locate on the walls of the house, in between the position of the windows and the ceiling as shown in Figure 1. The length of each ventilation hole varies according to the design concept, the size of the house. It could be observed that the most common size is about $1 \mathrm{~m}$ height and from $30 \mathrm{~cm}$ to $40 \mathrm{~cm}$ width.

However, noise problem associated with increases in number of motorcycles and automobiles is among the most serious environemntal issues that cities in the tropical developing countries are facing. Since the ventilation holes provide a direct pathway for noise to enter a residence, in many dwellings in those countries, glass panes have been found to be installed with ventilation holes and must be closed almost all day to deal temporarily with the situation. The initial design of ventilation holes is no longer appropriate with the current environment condition in urban areas of the tropical developing countries.

In this study, a model of ventilation hole device with soundproofing capability was proposed.
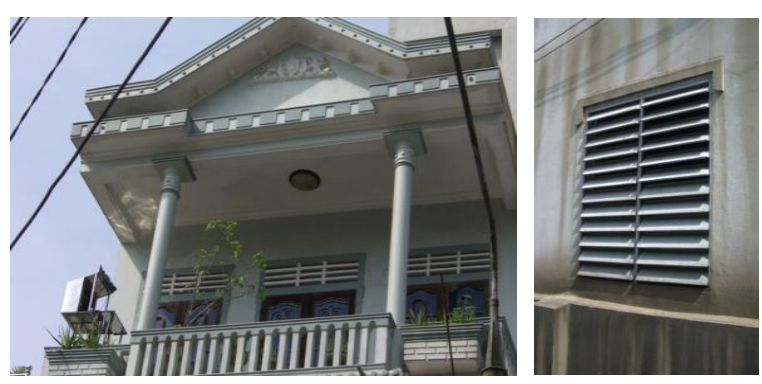

Figure1. The ventilation holes in tropical climates countries. 
The ventilation hole device is designed to have several input and output holes arranged on two sides of the rectangular chamber as shown in Figure 2. This device is installed into the wall with a common thickness of $20 \mathrm{~cm}$. According to the soundproofing principle, it is necessary to increase the volume or the height of the rectangular chamber, in addition to separating the position of the input and output, in order to enhance a soundproofing effect.

In this paper, the rectangle chamber was modeled as a piston-driven rectangular rigid tube with no losses and had large volume to attenuate the low-frequency traffic noise. General expression of an output pressure to a given input uniform volume velocity was obtained for the whole chamber by solving the wave equation.

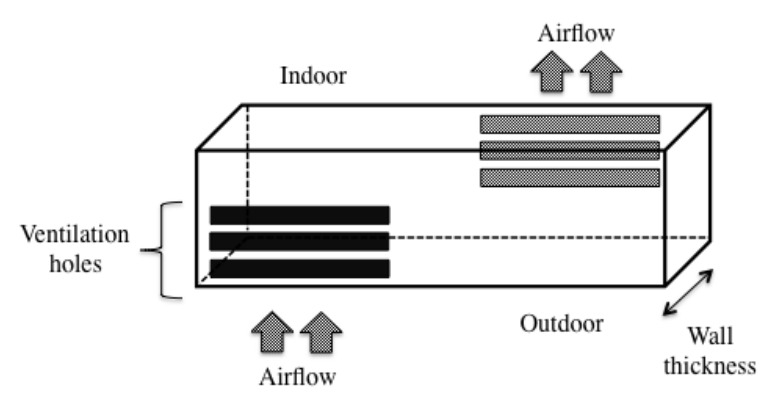

Figure2. The proposed ventilation hole device.

Since sound propagating through the chamber composed of plane and higher order mode waves which generate very high sound pressure levels, especially at resonance frequency, the soundproof capability could be maximized by preventing the generation of higher order mode waves.

\section{METHOD OF ANALYSIS}

The model of the rectangular soundproofing ventilation hole device has dimension $a \times b \times d$ as shown in Figure 3. The input and output are located on the opposite sides that have sectional areas of $S_{i}$ and $S_{0}$, respectively. For simplicity, only one input and one output are considered in this calculation.

Wave equation in terms of velocity potential $\Phi$ is given by[1]

$$
\left(\frac{\partial^{2}}{\partial x^{2}}+\frac{\partial^{2}}{\partial y^{2}}+\frac{\partial^{2}}{\partial z^{2}}\right) \Phi=\frac{1}{c^{2}} \frac{\partial^{2}}{\partial t^{2}} \Phi
$$

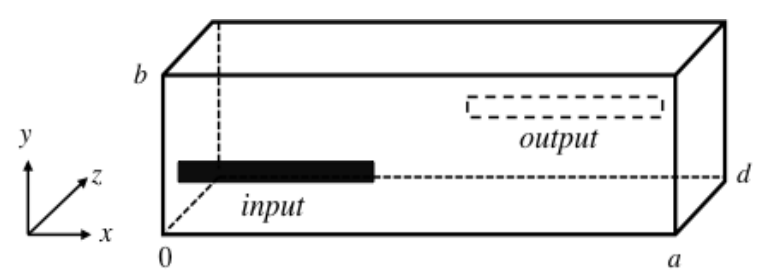

Figure3. Model of analysis and its co-ordinates

where $c$ is the sound velocity. Let $\Phi=\sqrt{2} \phi \exp (j \omega t)\left(j^{2}=-1, \omega=k c, k:\right.$ wave number ) then the general solution of (1) can be given as

$$
\begin{aligned}
& \phi=\left(A e^{\mu z}+B e^{-\mu z}\right)(C \sin (\alpha x)+D \cos (\alpha x)) \\
& \left(E \sin \left(\sqrt{s^{2}-\alpha^{2}} y\right)+F \cos \left(\sqrt{s^{2}-\alpha^{2}} y\right)\right)
\end{aligned}
$$

where $\mu^{2}=s^{2}-k^{2}, A, B, C, D, E$ and $\mathrm{F}$ are arbitrary constants determinable from the boundary conditions, other symbols are constants.

Assuming the walls of the cavity to be perfectly rigid and the loss at the wall can be neglected then boundary conditions are :

1) at $x=0,-\partial \phi / \partial x=0$

2) at $x=a,-\partial \phi / \partial x=0$

3) at $y=0,-\partial \phi / \partial y=0$

4) at $y=b,-\partial \phi / \partial y=0$

5) at $z=0,-\partial \phi / \partial z=V_{i} F_{i}(x, y)$

6) at $z=d, \quad-\partial \phi / \partial z=V_{0} F_{0}(x, y)$

where $-\partial \phi / \partial x,-\partial \phi / \partial y$ and $-\partial \phi / \partial z$ are the velocity component in the $x, y$ and $z$ directions, respectively. $V_{i}$ and $V_{0}$ are the driving velocity at the input and output, $F_{i}(x, y)$ and $F_{0}(x, z)$ are define as 1 on the area of inlet 
and outlet, zero at the other area of inlet and outlet, respectively.

In order to find $\phi$ from Eqs.(3) to ( 8), let $\phi_{a}$ be the solution of Eq.(2) obtained for the following boundary conditions:

1a) at $x=0,-\partial \phi_{a} / \partial x=0$

2a) at $x=a,-\partial \phi_{a} / \partial x=0$

3a) at $y=0,-\partial \phi_{a} / \partial y=0$

4a) at $y=b,-\partial \phi_{a} / \partial y=0$

5a) at $z=0,-\partial \phi_{a} / \partial z=V_{i} F_{i}(x, y)$

6a) at $z=d, \quad-\partial \phi_{a} / \partial z=0$

and let $\phi_{b}$ be the solution of Eq.(2) obtained for the following boundary conditions:

1b) at $x=0,-\partial \phi_{b} / \partial x=0$

2b) at $x=a,-\partial \phi_{b} / \partial x=0$

3b) at $y=0,-\partial \phi_{b} / \partial y=0$

4b) at $y=b,-\partial \phi_{b} / \partial y=0$

5b) at $z=0,-\partial \phi_{b} / \partial y=0$

6b) at $z=d, \quad-\partial \phi_{b} / \partial z=V_{0} F_{0}(x, y)$

then $\phi$ can be obtained as $\phi=\phi_{a}+\phi_{b}$.

$\phi$ is determined based on the boundary conditions (9) until (20). The sound pressure can be find by

$P=j k \rho c \phi$ and the average sound pressure of the outlet is calculated as following

$$
\begin{aligned}
& \bar{P}_{0}=\frac{1}{S_{0}} \iint_{S_{0}} P d x d y=j k Z_{w} \\
& \sum_{m=0}^{\infty} \sum_{n=0}^{\infty}\left\{\frac{\bar{Q}_{m, n}^{0, d}}{\mu_{m, n} \sinh \left(\mu_{m, n} d\right)} U_{i}+\frac{\cosh \left(\mu_{m, n} d\right) \bar{Q}_{m, n}^{d, d}}{\mu_{m, n} \sinh \left(\mu_{m, n} d\right)} U_{0}\right\}
\end{aligned}
$$

where

$$
\begin{aligned}
& \mu_{m, n}=\sqrt{(m \pi / a)^{2}+(n \pi / b)^{2}-k^{2}} \\
& \bar{Q}_{m, n}^{r, s}=\frac{4}{S_{r} S_{s}} \iint_{S_{r}} \cos \left(\frac{m \pi x}{a}\right) \cos \left(\frac{n \pi y}{b}\right) d x d y \\
& \times \iint_{S_{s}} \cos \left(\frac{m \pi x}{a}\right) \cos \left(\frac{n \pi y}{b}\right) d x d y
\end{aligned}
$$

$U_{i}=V_{i} S_{i}$ is an volume velocity supplied from the input and $U_{0}=V_{0} S_{0}$ is those of the output, $Z_{i}=\rho c / S_{i}$ is the characteristic impedance of the output.

The insertion-loss IL is given by [2]

$I L=10 \log _{10} \frac{W_{r}}{W_{0}}=20 \log _{10}|D|$

Where $W_{r}$ and $W_{0}$ are the radiated power at one point from the source and the radiated power from the output, respectively. The $D$ is four-poles parameter defined as

$$
D=\left.\frac{U_{i}}{U_{0}}\right|_{\bar{P}_{0}=0}
$$

Therefore, from (21) and (24)-(25) the Insertion Loss of ventilation hole device is

$$
\begin{aligned}
& I L=20 \log _{10}\left|\sum_{m=0}^{\infty} \sum_{n=0}^{\infty} \frac{\bar{Q}_{m, n}^{d, d}}{\bar{Q}_{m, n}^{0, d}} \cosh \left(\mu_{m, n} d\right)\right| \\
& =20 \log _{10}\left|\sum_{m=0}^{\infty} \sum_{n=0}^{\infty} \bar{Q}_{m, n} \cosh \left(\mu_{m, n} d\right)\right|
\end{aligned}
$$

where

$$
\bar{Q}_{m, n}=\frac{\iint_{S_{0}} \cos \left(\frac{m \pi x}{a}\right) \cos \left(\frac{n \pi y}{b}\right) d x d y}{\iint_{S_{i}} \cos \left(\frac{m \pi x}{a}\right) \cos \left(\frac{n \pi y}{b}\right) d x d y}
$$




\section{RESULTS AND DISCUSSION}

The average of the output sound pressure is derived from (21) in which the first term in the bracket represents the plane wave component, when $m=n=0$ and the others represents the higher-order mode wave components.

The low value of $\mathrm{Q}_{\mathrm{m}, \mathrm{n}}$ which appeared inside the bracket was selected to reduce the sound level of those waves to obtain a reliable soundproofing effect. It means that the reasonable positions and shapes of the input and the output were selected as shown in (23).

A calculation of insertion loss based on equation (26) is shown in Figure 4 where the ventilation hole device has dimension $\mathrm{a}=200 \mathrm{~cm}, \mathrm{~b}=40 \mathrm{~cm}$, $\mathrm{d}=20 \mathrm{~m}$, respectively. The input and output have shapes and positions as shown in Figure 5 . A black dotted line shown the IL of plane wave, the first term in the bracket of (26). The thick red line shows the IL of plane wave, higher order mode $(2,0),(0,1)$ and $(1,0)$. As an evidence from the figure, the insertion loss has a minus value at the resonance frequencies according to the result shown in the equation (21).

Insertion-loss value will be change depending on the input and output location and the shape of those holes.

An example is shown in Figure 6 and Figure 7. Soundproofing effect can be found to be high when the output is located at reasonable positions.

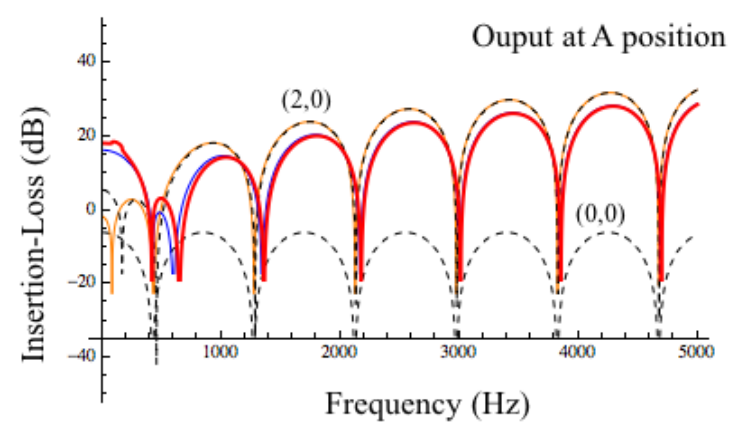

Figure4. Insertion Loss characteristics
Ouput at A position

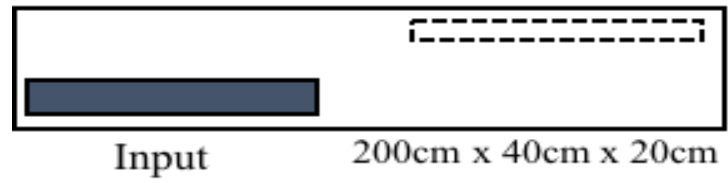

Figure5. Position of input and output when output is located at A position

Ouput at B position

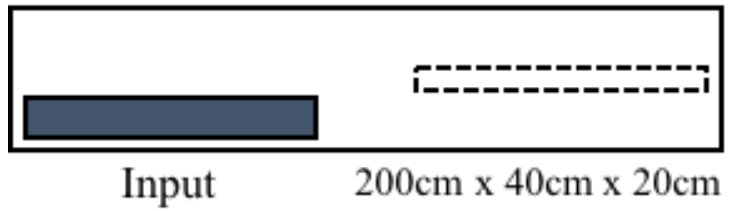

Figure6. Position of input and output when output is located at $B$ position

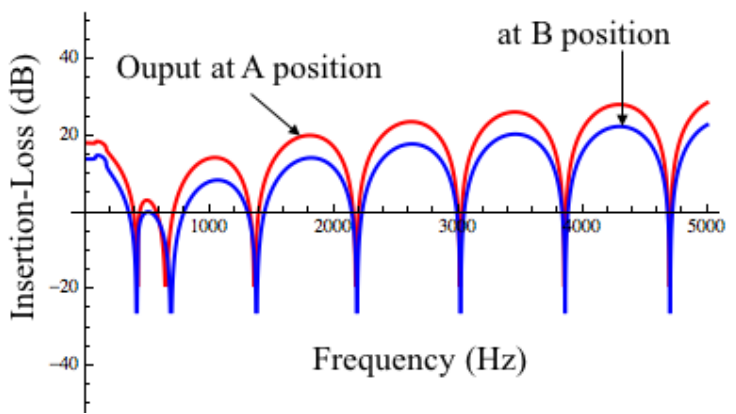

Figure7. Insertion Loss characteristics according to the output position

\section{CONCLUSION}

In this study, the calculation of sound propagation in the rectangular ventilation hole device with one inlet and one outlet was presented. The computation of the insertion-loss was based on solving the wave equation considering the higher order mode effects. Proposed formular is given by equation (26) for constant velocity volume and noise source. In order to improve the sound proofing capability of the device, the reasonable positions and shapes of input and output are required to be considered more thoroughly. The further findings will be presented in an upcoming report of this study.

\section{REFERENCES}

[1] Y. Nishimura, S. Nishimura, T. Nishimura and T. Yano "Sound propagation in soundproofing casement windows," Applied Acoustics, vol. 70, pp.1160-1167, 2009. 
[2] S.Nishimura and Y. Nishimura "Calculation Method for Predicting the Insertion-Loss of Soundproofing Ventilation Unit, "International Journal of Emerging Engineering Research and Technology, Vol. 4 , pp.32-37, 2016.

[3] S. Nishimura, T. Nishimura and T. Yano "Acoustic analysis of elliptical muffler chamber having a perforated pipe, " Journal of Sound and Vibration, vol. 297, pp.761-773, 2006.
[4] T. Nishimura and T. Ikeda "Four-poleparameters for an elliptical chamber with mean flow, "Electronics and Communication in Japan, vol. 81, pp.1-9, 1998.

[5] T. Nishimura, T. Ando and T. Ikeda"Resonance of elliptical muffler chamber having a non uniformly perforated pipe, "Electronics and Communication in Japan, vol.85, pp. 22-28, 2002.

Citation: Y. Nishimura, S. Nishimura, T. Nguyen and K. Dang, "Calculation of Sound Propagation in Ventilation Hole Device", International Journal of Emerging Engineering Research and Technology, vol. 5, no. 4, pp. 1-5, 2017.

Copyright: (C) 2017 Y. Nishimura, et al. This is an open-access article distributed under the terms of the Creative Commons Attribution License, which permits unrestricted use, distribution, and reproduction in any medium, provided the original author and source are credited. 